\title{
Correlation of Quantitative Changes in Bone Marrow Lymphocyte Subpopulations with Some Rhabdomyosarcoma Prognosis Factors in Children
}

\author{
Olga P. Kolbatskaya1, Tatiana V. Gorbunova1, Svetlana V. Chulkova1,2, Anna A. Khachatryan³, \\ Nikolai N. Tupitsyn", Vladimir G. Polyakov ${ }^{1,3}$ \\ ${ }^{1}$ National Medical Research Center of Oncology Named after N.N. Blokhin, Ministry of Health of Russia, Moscow, Russia \\ ${ }^{2}$ Medical Faculty of the Oncology and Radiotherapy, Pirogov Russian National Research Medical University, Ministry of Health of \\ Russia, Moscow, Russia \\ ${ }^{3}$ Russian Medical Academy of Continuing Professional Education, Ministry of Health of Russia, Moscow, Russia \\ Email: helgaopk69@yandex.ru,nntca@yahoo.com
}

How to cite this paper: Kolbatskaya, O.P., Gorbunova, T.V., Chulkova, S.V., Khachatryan, A.A., Tupitsyn, N.N. and Polyakov, V.G. (2021) Correlation of Quantitative Changes in Bone Marrow Lymphocyte Subpopulations with Some Rhabdomyosarcoma Prognosis Factors in Children. Advances in Biological Chemistry, 11, 234-250. https://doi.org/10.4236/abc.2021.115016

Received: July 2, 2021

Accepted: October 26, 2021

Published: October 29, 2021

Copyright $\odot 2021$ by author(s) and Scientific Research Publishing Inc. This work is licensed under the Creative Commons Attribution International License (CC BY 4.0).

http://creativecommons.org/licenses/by/4.0/

\section{(c) (i) Open Access}

\begin{abstract}
Rationale: The known prognosis factors for rhabdomyosarcoma (RMS) in children do not always explain the unsatisfactory outcome of treatment. Changes in the subpopulation composition of Bone Marrow (BM) effector cells during the development of RMS may indicate new directions for the search for prognostic factors and points for the impact of targeted therapy. Purpose: To identify correlations between quantitative changes in the levels of subpopulations of T, B and NK-lymphocytes of BM and known risk factors for RMS in children. Objects: The study included 31 patients. The main group included 16 patients with RMS, average age $-6.8 \pm 1.0$ years, while children $1-10$ years old $-13(81.3 \%)$, over 10 years old-3 (18.8\%) people, girls and boys were 8 people each. The embryonic variant of RMS was established in $10(62.5 \%)$ cases, the alveolar variant-in $4(25 \%)$ cases, in two patients (12.5\%), the histological variant was not established. In 12 (75\%) patients, an unfavorable localization of the RMS (parameningeal, extremities, prostate, bladder) was revealed, in 4 patients $(25 \%)$, the localization of the tumor was regarded as favorable. Patients with $\mathrm{T} 2 \mathrm{~b}-13(81.2 \%)$ and $\mathrm{T} 2 \mathrm{a}-2(12.5 \%)$ stages prevailed. Regional and distant metastases were detected in $10(52.6 \%)$ patients. The comparison group included 15 children in whom the presence of malignant neoplasia was excluded, the average age was $8.4 \pm 1.5$ years, 11 boys (73.7\%) and 4 girls (26.3\%). Methods: All patients underwent morphological (myelogram counting) and immunological (quantitative analysis of lymphocytic subpopulations)
\end{abstract}


bone marrow studies. Immunophenotyping in all patients was carried out by direct immunofluorescence using a triple fluorescent label. Results: Significant differences in the levels of subpopulations of BM T-lymphocytes were found when comparing the values of the main group, distributed by localization and histological variant, with the data obtained in the control group of patients. For example, the percentage of $\mathrm{CD} 3+\mathrm{T}$ cells with the co-stimulatory molecule CD28+ was significantly higher in patients with parameningeal RMS $(\mathrm{p}=0.010)$. Conclusion: Each clinical group of patients has its own individual immunological characteristics. The results obtained by us can be considered indicative and regarded as starting points for further study of the peculiarities of the subpopulation composition of BM in patients with RMS.

\section{Keywords}

Rhabdomyosarcoma, Bone Marrow, Children, Lymphocyte Subpopulations

\section{Introduction}

\subsection{Justification}

Rhabdomyosarcoma (RMS) is a soft tissue sarcoma, which accounts for 3\% - 5\% of all solid tumors of childhood, characterized by rapid progression, lymphogenous and hematogenous metastasis [1] [2]. A comprehensive approach is used to treat patients with RMS, which includes chemotherapy, radiation therapy, and surgery. When using this method, the 5-year overall survival rate for RMS reaches $65.4 \%$, and when distant metastases are detected simultaneously with the primary tumor, this indicator does not exceed $35.7 \%$ [3].

The choice of treatment tactics is based on identifying factors of poor prognosis, which include: age over 10 years, unfavorable localization and size of the primary tumor over $5 \mathrm{~cm}$, alveolar histological variant, lymph node involvement, and the presence of regional and distant metastases [2]. The influence of each factor on the outcome of the disease has been proven throughout the IRS I-IV studies (1972-1997) [1] [2]. Later, genetic markers of the unfavorable course of RMS were identified, for example, the chimeric PAX-3FKHR gene [1] [2]. But the known prognostic factors do not always explain the unsatisfactory outcome of treatment of individual patients after standard therapy within the same risk group [1] [2]. Therefore, simultaneously with the improvement of anticancer therapy, the search for new prognosis criteria, including immunological ones, continues.

One of the possible ways to improve survival rates may be the identification of clinically effective agents that affect RMS-specific targets [1]. Another direction of the search for "targets" for immunotherapy is directed towards the study of the functions of immunocompetent cells and their interaction with the tumor. BM is a key organ in the formation of an antitumor immune response in the develop- 
ment of hematogenously spreading neoplasias, which include RMS [4]. BM contains the main antibody-producing cells (plasma cells) and effector cells (Cytotoxic T-Lymphocytes (CTLs)), T-helpers, regulatory $\mathrm{T}$ cells, dendritic cells, natural killer cells, natural killer $\mathrm{T}$ cells, myeloid suppressor and mesenchymal stem cells [5] [6]. The main role in the antitumor immune response is played by CTLs, which are formed from a naive T-lymphocyte after antigen presentation, recognition of antigenic determinants by the T Cell Receptor (TCR), subsequent proliferation, and clone formation of antigen-specific CD3+ CD8+ CTLs [6]. It was experimentally proved that in the absence of secondary lymphoid organs, $\mathrm{BM}$ macrophages are able to present an antigen to naive T-lymphocytes with the subsequent initiation of primary $\mathrm{T}$ cell reactions and the formation of clones of antigen-specific CTLs and memory cells and to exercise antitumor immunity, the effectiveness of which directly correlates with the number of mature $\mathrm{T}$ cells [4].

But, on the other hand, BM cells, such as Mesenchymal Stem Cells (MSC) and regulatory T-lymphocytes, have recently been identified as cells that contribute to the metastasis of a malignant tumor. The innate versatility of these cells provides a variety of functional possibilities for the development of neoplasias, ranging from structural support to signal-mediated suppression of the body's immune response [7].

Defects of the BM immune system are of decisive importance at the stage of the onset of small round cell sarcomas, since a number of authors prove the multipotent BM mesenchymal stem cell as a source of origin of the tumor clone [8]. In addition to participating in the immune response, $\mathrm{BM}$ is an immunological "niche" in micrometastatic lesions, since it creates optimal conditions in which the tumor cell safely, avoids the effects of chemoradiation therapy being in a dormant state, and is a further source of disease recurrence [9].

Despite the wide recognition of the significance of the experimental work of Feuerer et al. and the validity of their conclusions, this study raises many questions in the aspect of studying the antitumor function of $\mathrm{BM}$ in various malignant tumors and the possibility of clinical application of the data obtained. On the one hand, attempts to conduct immunotherapy for sarcomas have so far been unsuccessful [10]. On the other hand, the possibility of revealing changes in the subpopulation composition of BM effector cells during the development of RMS in children (as a manifestation of immunodeficiency), may indicates new directions for searching for prognostic factors and points for the impact of targeted therapy.

\subsection{Purpose of the Study}

To reveal the correlations between the quantitative changes in the levels of subpopulations of T-lymphocytes-effectors of the bone marrow and the known risk factors for RMS in children. 


\subsection{Study Type}

The research was a retrospective cohort non-randomized study.

\subsection{Study Period}

The research was carried out in 2004-2011. The analysis of the results was carried out as of 01.01.2019.

\subsection{Study Inclusion Criteria}

1) Patients under 17 years of age who have a morphological diagnosis of rhabdomyosarcoma for the first time;

2) Informative morphological and immunological analyzes of BM, obtained before the start of the special study;

3) The established stage of RMS according to the data of the complex examination, which included: MRI/CT of the primary tumor, CT of the chest organs, radioisotope diagnostics (RID) of bones and soft tissues, ultrasound of the areas of regional metastasis and the abdominal organs;

4) Parental consent to participate in the study;

5) To include patients in the control group, a necessary condition was the absence of data on the presence of a malignant tumor, confirmed by the results of a comprehensive examination and/or morphological study.

\subsection{Exclusion Criteria}

1) Lack of informed consent of the patient's parents for bone marrow examination;

2) Absence of myelogram in the medical history;

3) Non-informative myelogram (significant admixture of peripheral blood in the bone marrow punctate);

4) Total replacement of hematopoietic tissue with tumor cells.

\subsection{Research Objects}

The study included 31 patients. The average age at the time of the study was 6.5 years ( 1 - 17 years), boys $-19(61.3 \%)$, girls-12 (38.7\%).

\subsection{Main Group}

The main group included 16 patients with RMS, aged from 1 to 15 years, with an average age of $6.8 \pm 1.0$ years, girls and boys were 8 people each. RMS was more often detected in children $1-10$ years old -13 patients $(81.2 \%)$. The clinical data of patients with RMS included in our study are presented in Table 1.

As can be seen from the data presented in Table 1, the majority of patients included in our study (12 people, $75 \%$ ) had an unfavorable localization of the primary tumor and, accordingly, a poor prognosis of the disease.

According to the morphological study, the prognostically favorable embryonic variant of RMS was also found in the majority of patients (10 people, 62.5\%), 
Table 1. The clinical data of patients with RMS.

\begin{tabular}{cccccccc}
\hline № & Initials & Sex & Age & $\begin{array}{c}\text { The nature of the } \\
\text { tumor localization }\end{array}$ & $\begin{array}{c}\text { Localization of } \\
\text { the tumor }\end{array}$ & $\begin{array}{c}\text { Histological } \\
\text { variant }\end{array}$ & TNM \\
\hline 1 & D. B. & F & 2 & Unfavorable & Paravertebral & Alveolar & T2aN0M0 \\
2 & D. D. & M & 8 & Unfavorable & Parameningeal & Embryonic & T2bN0M0 \\
3 & E. D. & M & 6 & Unfavorable & Parameningeal & Embryonic & T2bN0M0 \\
4 & Z. A. & M & 2,6 & Favorable & Head and neck & Embryonic & T2bN1M0 \\
5 & I. M. & M & 13 & Unfavorable & Parameningeal & Embryonic & T2bN1M1 \\
6 & K. N. & F & 6 & Favorable & Pelvis & Embryonic & T2bN0M0 \\
7 & K. D. & F & 11 & Unfavorable & Extremity & Alveolar & T1aN0M0 \\
8 & K. A. & F & 4 & Unfavorable & Parameningeal & Alveolar & T1bN1M1 \\
9 & L. S. & F & 4 & Unfavorable & Extremity & Alveolar & T2bN0M0 \\
10 & L. P. & M & 15 & Unfavorable & Prostate & Embryonic & T2bN0M1 \\
11 & L. D. & F & 7 & Unfavorable & Parameningeal & Not established & T2bN1M1 \\
12 & M. N. & F & 1 & Unfavorable & Parameningeal & Embryonic & T2bN0M0 \\
13 & S. B. & F & 7 & Favorable & Orbit & Embryonic & T2aN0M0 \\
14 & H. M. & F & 8 & Favorable & Pelvis & Not established & T2bN1M1 \\
15 & Y.S. & M & 7 & Unfavorable & Parameningeal & Embryonic & T2bN0M0 \\
16 & U. Ya. & M & 5 & Unfavorable & Parameningeal & Embryonic & T2bN1M0 \\
\hline
\end{tabular}

and the unfavorable alveolar variant in $4(25 \%)$ cases; in $2(12.5 \%)$ patients the histological variant was not established. In 4 patients (25\%) the unfavorable localization of the primary tumor was combined with its unfavorable histological variant. On the contrary, in 3 patients (18.8\%) with a favorable localization of the tumor, its favorable histological variant was diagnosed. In 7 patients (43.8\%) with unfavorable localization of the primary tumor, its histological variant was prognostically favorable.

The prevalence of the tumor process was assessed on the basis of the data of radioisotope studies of bones and soft tissues, ultrasound of soft tissues and regional lymph nodes, $\mathrm{X}$-ray examination, $\mathrm{CT}$ of the chest and anatomical zones in which metastatic lesions were suspected. When assessing the size of the tumor, it was revealed that patients with $\mathrm{T} 2 \mathrm{~b}-13(81.2 \%)$ stage prevailed, T1a and $\mathrm{T} 2 \mathrm{a}-$ isolated cases. The presence of metastases (regional and distant) was diagnosed in $5(32.5 \%)$ patients, in $10(62.5 \%)$ patients there was no lymph node in the disease debut, a combination of distant metastases and lymphatic nodes lesions were detected in 4 (25\%) patients.

\subsection{Control Group}

Considering the insufficient amount of information in the medical literature on the number of BM lymphocyte subpopulations in normal children, we formed a control group to compare with the data obtained in the study of the levels of 
these cells in patients with RMS. The comparison group included 15 children aged 1 to 17 years, 11 boys (73.7\%) and 4 girls (26.3\%) with an average age of 8.4 \pm 1.5 years. Patients were examined in the scientific advisory department of the Scientific Research Institute of pediatric oncology and hematology in order to confirm/exclude the presence of malignant neoplasia. During the examination, the following diagnoses were established: benign tumors, malformations of organs and tissues, and inflammatory processes. The presence of a malignant process was excluded according to a comprehensive examination, including histological examination.

\section{Research Methods}

The diagnosis of RMS in all cases was verified according to the results of morphological and/or immunohistochemical studies. In all patients included in our work, bone marrow punctate in the amount of $0.5 \mathrm{ml}$ of BM was taken in test tubes with dry EDTA, followed by the preparation of morphological preparations and their staining according to Pappenheim. All patients underwent morphological (myelogram counting) and immunological (quantitative analysis of lymphocytic subpopulations) BM studies. The panel of monoclonal antibodies (MCA) used in the flow cytometric diagnosis included antibodies to the total leukocyte antigen CD45. Immunophenotyping in all patients was carried out by direct immunofluorescence using a triple fluorescent label. Nucleated BM punctate cells were isolated by standard erythrocyte lysis using FACS Lysing solution, BD. Staining of lymphocytic populations was carried out using a wide panel of monoclonal antibodies MCA (tricolor fluorescence), manufacturer-Becton Dickinson, USA. The panel of antibodies used to study subpopulations of BM lymphocytes in patients with RMS is presented in Table 2.

Table 2. MCA panel for studying subpopulations of BM lymphocytes in patients with RMS.

\begin{tabular}{ccccc}
\hline Sample & FITC & PE & PE/Cy5 (PerCP) & Note \\
\hline 1 & Ig G2a & IgG1 & IgG1 & Control \\
2 & Ig G2a & IgG1 & CD45 & Control \\
3 & CD3 & CD56 & CD45 & T cells u NK-cells \\
4 & CD19 & CD5 & CD45 & B-lymphocytes \\
5 & IgG2a & IgG1 & CD3 & Control within T cells \\
6 & CD4 & CD62L & CD3 & T-helpers, incl. CD62L \\
7 & CD8 & CD38 & CD3 & CTL, incl. CD38 \\
8 & CD8 & HLA-DR & CD3 & CTL, incl. HLA-DR+ \\
9 & CD57 & CD8 & CD3 & CTL, incl. CD57 \\
10 & CD8 & CD28 & CD3 & CTL, incl. CD28 \\
11 & TCR $\alpha \beta$ & CD25 & CD3 & TCR $\alpha \beta$ \\
12 & TCR $\gamma \delta$ & CD2 & CD3 & TCR $\gamma \delta$ \\
\hline
\end{tabular}


When studying subpopulations of lymphocytes from patients in the control group, the same panel of antibodies was used as in the study of BM lymphocytes from patients with RMS (see Table 2).

Statistical calculations were performed using the SPSS 17 software and included correlation analysis (Pearson's coefficient), comparison of means (Student's test) and Fisher's exact test with a confidence interval of $95 \%$. Differences were considered statistically significant at $\mathrm{p}<0.05$ (95\% accuracy).

\section{Results}

We compared the results of the study of the level of BM lymphocytes subpopulations obtained in the main group of patients with the results in the control group and correlated them with known risk factors for RMS.

\subsection{Comparison of the Number of Subpopulations of BM Lymphocytes in All Patients with the Results of the Control Group}

We compared the results of the study of the number of subpopulations of lymphocytes BM in the all group of patients and the data of the control group and found that the relative content of $\mathrm{CD} 3+$ subpopulations was increased in patients with RMS (in particular, CD8+ subpopulations) with the activation molecule HLA-DR+ $(\mathrm{p}=0.003$ and $\mathrm{p}=0.001$ respectively) was increased in patients with RMS as well as T cells with a $\gamma \delta$ T cell receptor $(\mathrm{p}=0.013)$ (Table 3$)$. The absolute amounts of these subpopulations did not differ from the control values $(\mathrm{p}>$ $0.050)$.

It was also found that the percentage of CD4+ T cells with the Leu8+ adhesion molecule in patients with RMS was reduced compared to the control group ( $\mathrm{p}=$ 0.015) (Table 4). On the contrary, the absolute content of these cells in the BM of patients with RMS was higher compared to the control value $(\mathrm{p}=0.047)$ (Table 4).

Table 3. Comparison of relative content CD3+ HLADR+, CD8+ HLADR+ and $\gamma \delta T C R T$ cells of BM in all patients with RMS with the data of the control group.

\begin{tabular}{cccc}
\hline Subpopulations (\%) & All patients with RMS & Control group & $\mathrm{P}$ \\
\hline CD3+ HLADR+ & $35.5 \pm 6.0$ & $12.5 \pm 2.0$ & 0.003 \\
& $(\mathrm{n}=15)$ & $(\mathrm{n}=12)$ & \\
CD8+ HLADR+ & $28.7 \pm 6.1$ & $8.9 \pm 1.4$ & 0.001 \\
& $(\mathrm{n}=10)$ & $(\mathrm{n}=11)$ & \\
$\gamma \delta$ TCR T cells & $15.7 \pm 1.6$ & $9.6 \pm 1.5$ & 0.013 \\
\end{tabular}

Table 4. Comparison of the relative and absolute number of Leu8+ CD4+ T cells in all patients with RMS with the control group.

\begin{tabular}{cccc}
\hline Subpopulation & All patients with RMS & Control group & $\mathrm{P}$ \\
\hline Leu8+ CD4+ (\%) & $\begin{array}{c}17.6 \pm 3.6 \\
(\mathrm{n}=10)\end{array}$ & $\begin{array}{c}33.5 \pm 4.7 \\
(\mathrm{n}=10)\end{array}$ & 0.015 \\
Leu8+ CD4+ (abs.) & $\begin{array}{c}7.2 \pm 1.3 \\
(\mathrm{n}=6)\end{array}$ & $\begin{array}{c}5.3 \pm 1.1 \\
(\mathrm{n}=10)\end{array}$ & 0.047 \\
\hline
\end{tabular}




\subsection{Interrelation between the Number of BM Lymphocyte Subpopulations in Patients with RMS and the Localization of the Primary Tumor}

As you know, the localization of the primary tumor is of great clinical and prognostic significance. We found that in patients with favorable tumor localization the absolute content of CD3+ HLADR+, CD8+ HLADR+ subpopulations was significantly higher ( $\mathrm{p}=0.047,0.023$ and 0.048 , respectively) (Table 5). The relative amounts of these subpopulations did not differ from the control values ( $p>$ $0.050)$.

Comparison of the relative and absolute content of BM T-lymphocytes in patients with RMS with an unfavorable prognostic tumor localization and data from the control group revealed an increase in the BM of patients with RMS in the percentage of CD3+ HLADR+ $(\mathrm{p}=0.001), \mathrm{CD} 8+\mathrm{HLADR}+(\mathrm{p}=0.001)$ subpopulations and T-lymphocytes with the $\gamma \delta \mathrm{TCR}$ receptor $(\mathrm{p}=0.013)$, and a decrease in the percentage of T-helper cells $(\mathrm{CD} 3+\mathrm{CD} 4+$ cells $)(\mathrm{p}=0.046)$, in particular T-helpers with the adhesion molecule Leu8+ (Leu8+ CD4+ cells) $(\mathrm{p}=0.017)$ (Table 6). The absolute numbers of the listed $T$ cell subpopulations did not differ from the results in the control group ( $\mathrm{p}>0.05)$.

Separately we compared the results of the study of patients with unfavorable and favorable localization of the primary tumor, and we revealed significant differences in the absolute content of a number of subpopulations of BM T cells. The comparison results are shown in Table 7.

Table 5. Comparison of the absolute content of CD3+ HLADR+ and CD8+ HLADR+ $\mathrm{BM}$ subpopulations of patients with RMS (favorable localization of the primary tumor) and the data of the control group.

\begin{tabular}{cccc}
\hline $\begin{array}{c}\text { Subpopulations } \\
\text { (absolute numbers) }\end{array}$ & $\begin{array}{c}\text { Patients with RMS } \\
\text { (favorable localization } \\
\text { of the primary tumor) }\end{array}$ & Control group & $\mathrm{P}$ \\
\hline CD3+ HLADR+ & $5.5 \pm 1.8(\mathrm{n}=3)$ & $2.5 \pm 0.6(\mathrm{n}=11)$ & 0.047 \\
CD8+ HLADR+ & $4.4 \pm 1.6(\mathrm{n}=2)$ & $1.8 \pm 0.4(\mathrm{n}=10)$ & 0.048 \\
\hline
\end{tabular}

Table 6. Comparison of the relative content of CD3+ HLADR+, CD8+ HLADR+, CD3+ CD4+, Leu8+ CD4+, $\gamma \delta$ TCR-T cells of BM of patients with RMS (unfavorable tumor localization) and the control group.

\begin{tabular}{cccc}
\hline Subpopulations (\%) & $\begin{array}{c}\text { Patients with RMS (unfavorable } \\
\text { tumor localization) }\end{array}$ & Control group & $\mathrm{P}$ \\
\hline CD3+ HLADR+ & $40.1 \pm 6.9(\mathrm{n}=12)$ & $12.5 \pm 2.0(\mathrm{n}=12)$ & 0.001 \\
CD8+ HLADR+ & $32.5 \pm 7.1(\mathrm{n}=8)$ & $8.9 \pm 1.4(\mathrm{n}=11)$ & 0.001 \\
CD3+ CD4+ & $30.3 \pm 3.1(\mathrm{n}=11)$ & $39.3 \pm 2.8(\mathrm{n}=14)$ & 0.046 \\
Leu8+ CD4+ & $16.2 \pm 4.3(\mathrm{n}=8)$ & $33.5 \pm 4.7(\mathrm{n}=10)$ & 0.017 \\
$\gamma \delta$ TCR-T cells & $16.7 \pm 2.1(\mathrm{n}=8)$ & $9.6 \pm 1.5(\mathrm{n}=10)$ & 0.013 \\
\hline
\end{tabular}


Table 7. Comparison of the absolute number of subpopulations of BM lymphocytes in patients with RMS with favorable and unfavorable localization of the primary tumor.

\begin{tabular}{cccc}
\hline $\begin{array}{c}\text { Subpopulations } \\
\text { (absolute quantities) }\end{array}$ & $\begin{array}{c}\text { Patients with RMS } \\
\text { (favorable localization } \\
\text { of the primary tumor) }\end{array}$ & $\begin{array}{c}\text { Patients with RMS } \\
\text { (unfavorable localization } \\
\text { of the primary tumor) }\end{array}$ & P \\
\hline CD3+ & $19.5 \pm 2.4(\mathrm{n}=3)$ & $6.4 \pm 1.9(\mathrm{n}=10)$ & 0.006 \\
CD3+ CD2+ & $29.5 \pm 3.0(\mathrm{n}=3)$ & $9.5 \pm 3.8(\mathrm{n}=7)$ & 0.013 \\
CD3+ CD8+ & $16.3 \pm 2.2(\mathrm{n}=3)$ & $5.4 \pm 1.4(\mathrm{n}=9)$ & 0.003 \\
CD5+ & $19.8 \pm 2.5(\mathrm{n}=3)$ & $5.9 \pm 2.0(\mathrm{n}=9)$ & 0.047 \\
CD56+ & $3.4 \pm 0.5(\mathrm{n}=3)$ & $0.8 \pm 0.2(\mathrm{n}=8)$ & 0.000 \\
CD19+ CD5+ & $16.2 \pm 11.9(\mathrm{n}=3)$ & $0.8 \pm 0.4(\mathrm{n}=8)$ & 0.044 \\
$\alpha \beta$ TCR & $24.9 \pm 1.6(\mathrm{n}=3)$ & $8.2 \pm 3.5(\mathrm{n}=7)$ & 0.018 \\
$\gamma \delta$ TCR & $4.1 \pm 0.8(\mathrm{n}=3)$ & $1.3 \pm 0.4(\mathrm{n}=7)$ & 0.005 \\
CD8+ HLADR+ & $4.4 \pm 1.6(\mathrm{n}=2)$ & $1.7 \pm 0.3(\mathrm{n}=7)$ & 0.017 \\
\hline
\end{tabular}

Based on the data presented in Table 7, it is shown that in BM of patients with RMS with a favorable prognostic localization of the primary tumor, the absolute numbers of $\mathrm{CD} 3+, \mathrm{CD} 3+\mathrm{CD} 2+, \mathrm{CD} 3+\mathrm{CD} 8+, \mathrm{CD} 5+, \mathrm{CD} 56+, \mathrm{CD} 19+\mathrm{CD} 5+$, $\mathrm{CD} 8+$ HLADR+ subpopulations of lymphocytes $(\mathrm{p}=0.006,0.013,0.003,0.047$, $0.000,0.044$ and 0.017 , respectively) are significantly higher and the absolute content of T cells with $\alpha \beta \mathrm{TCR}$ and $\gamma \delta \mathrm{TCR}$ T cell receptors is increased $(\mathrm{p}=0.018$ and 0.005 , respectively). The absolute content of CD3+ CD4+ T cells and CD4/CD8 ratio between the compared groups did not differ $(p>0.050)$. The relative content of all compared subpopulations of BM lymphocytes in RMS patients of the two prognostic groups (with favorable and unfavorable tumor localization) was not significantly differed ( $\mathrm{p}>0.050$ ).

During the analysis, we noted that the number of $\mathrm{T}$ cell subpopulations of $\mathrm{BM}$ in patients with RMS with a parameningeal tumor localization $(n=8)$ differed from the results of RMS patients with various other localizations of the primary tumor. In patients with RMS of parameningeal localization the percentage of CD3+ $\mathrm{T}$ cells with the co-stimulatory molecule CD28+ was significantly higher $(60.3 \%$ $\pm 5.9 \%$ versus $14.0 \% \pm 12.4 \% \mathrm{p}=0.010$ ), however, the absolute number of CD3+ CD28+ T cells did not differ $(\mathrm{p}=0.610)$.

\subsection{Interrelation of Histological Variant of RMS with the Quantitative Composition of Subpopulations of Mature BM T-Lymphocytes}

We also found that, depending on the histological variant of RMS (alveolar or embryonic), there are significant differences in the relative number of $\mathrm{T}$ cells, $\mathrm{Ta}$ ble 8 and Table 9. To identify correlations, we compared the data of patients with alveolar RMS and the control group. 
Table 8. Comparison of the relative number of CD3+ HLADR+, CD3+ CD4+, CD3+ CD28+, CD8+ HLADR,$+ \gamma \delta$ TCR of BM T-lymphocytes in patients with alveolar RMS with the data of the control group.

\begin{tabular}{cccc}
\hline Subpopulations (\%) & Patients with alveolar RMS & Control group & $P$ \\
\hline CD3+ HLADR+ & $47.7 \pm 13.0(\mathrm{n}=4)$ & $12.5 \pm 2.0(\mathrm{n}=15)$ & 0.014 \\
CD8+ HLADR+ & $25.2 \pm 15.0(\mathrm{n}=3)$ & $8.9 \pm 1.4(\mathrm{n}=11)$ & 0.049 \\
CD3+ CD4+ & $24.8 \pm 3.9(\mathrm{n}=4)$ & $39.3 \pm 2.8(\mathrm{n}=14)$ & 0.023 \\
CD3+ CD28+ & $15.3 \pm 12.6(\mathrm{n}=3)$ & $60.5 \pm 9.8(\mathrm{n}=11)$ & 0.046 \\
$\gamma \delta$ TCR T cells & $20.4 \pm 3.8(\mathrm{n}=3)$ & $9.6 \pm 1.5(\mathrm{n}=10)$ & 0.008 \\
\hline
\end{tabular}

Table 9. Comparison of the relative number of CD3+ HLADR+, CD8+ HLADR+, Leu8+ CD4+ T-lymphocytes and $\gamma \delta \mathrm{TCR}$ T-lymphocytes of BM in patients with embryonic RMS with the data of the control group.

\begin{tabular}{cccc}
\hline Subpopulations (\%) & Patients with embryonic RMS & Control group & $\mathrm{P}$ \\
\hline CD3+ HLADR+ & $40.6 \pm 7.4(\mathrm{n}=9)$ & $12.5 \pm 2.0(\mathrm{n}=12)$ & 0.001 \\
CD8+ HLADR+ & $38.6 \pm 6.2(\mathrm{n}=5)$ & $8.9 \pm 1.4(\mathrm{n}=11)$ & 0.000 \\
Leu8+ CD4+ & $12.6 \pm 3.3(\mathrm{n}=5)$ & $33.5 \pm 4.7(\mathrm{n}=10)$ & 0.011 \\
$\gamma \delta$ TCR & $15.5 \pm 1.5(\mathrm{n}=6)$ & $9.6 \pm 1.5(\mathrm{n}=10)$ & 0.020 \\
\hline
\end{tabular}

In patients with alveolar RMS, compared with the control group, the percentage of $\mathrm{CD} 3+\mathrm{CD} 4+(\mathrm{p}=0.023), \mathrm{CD} 3+\mathrm{T}$ cells with the co-stimulatory molecule $\mathrm{CD} 28+(\mathrm{p}=0.046)$ is significantly reduced and higher percentages of CD3+ HLA$\mathrm{DR}+\mathrm{T}$ cells $(\mathrm{p}=0.014)$ and CD8+ CTLs with an activation molecule HLADR+ $(\mathrm{p}=0.049)$ and $\mathrm{T}$ cells with a $\gamma \delta \mathrm{TCR}$ receptor $(\mathrm{p}=0.008)($ Table 8$)$.

Comparison of the relative and absolute content of BM T-lymphocytes in patients with embryonic RMS with the data of the control group also revealed an increase in the percentage of CD3+ HLADR+ $(\mathrm{p}=0.001), \mathrm{CD} 8+\mathrm{HLADR}+(\mathrm{p}=$ $0.000), \gamma \delta$ TCR-T cells $(\mathrm{p}=0.020)$ and a decrease percentage of Leu8+ CD4+ T cells $(\mathrm{p}=0.011)$; the absolute amounts of these subpopulations did not differ from the control values $(p>0.050)$ (Table 9).

Comparison of the relative and absolute content of subpopulations of BM lymphocytes in patients with RMS between alveolar and embryonic histological variants did not reveal significant differences ( $p>0.050$ ).

\section{Discussion}

In this work, we made an attempt to identify the features of the subpopulation composition of BM lymphocytes in pediatric RMS patients. Since BM is a central immunoregulatory organ, in which the development of solid tumors leads to a change in the composition of T-effector cells that carry out an antitumor immune response, the efficiency of which directly correlates with the number of mature BM T cells, the changes in the number of mature T-lymphocytes can directly 
correlate with known risk factors for RMS or supplement them [5].

We found that the relative number of activated CD3+ HLADR $+\mathrm{T}$ cells was significantly increased in all patients with RMS compared to the control group (due to an increase in the percentage of activated CD8+ HLADR+ CTL), but no increase in the absolute number of these subpopulations was observed. These results may indicate that the proliferative response to the recognition of sarcoma antigens by $\mathrm{T}$ cells is moderate due to the low immunogenicity of RMS [11]. The decrease in the relative and absolute content of another $\mathrm{T}$ cell subpopulationT-helper cells with an adhesion molecule (Leu8+ CD4+ cells) may reflect the immunodeficiency state of the patient's body during the development and proliferation of RMS due to the suppression of the cellular link of immunity by direct or indirect exposure to the tumor.

While the majority of mature $\mathrm{T}$ lymphocytes express the $\alpha \beta \mathrm{T}$ cell receptor ( $\alpha \beta$ TCR heterodimer), a small proportion of T cells express an alternative $\gamma \delta \mathrm{TCR}$ heterodimer, through which it recognizes various MHC-like stress-induced auto-antigens expressed by malignant cells without primary antigen presentation in combination with the MHC molecule [6]. The functions of $\gamma \delta$ TCR-T cells have not yet been fully studied, although at present the prevailing opinion is that they serve as one of the connecting components between innate and acquired immunity and are one of the first barriers to pathogens [6]. Thus, $\gamma \delta \mathrm{TCR} \mathrm{T}$ cells express some markers characteristic of NK cells (NKG2D) and antigen presenting cells-CD16 and TLR. In addition, these T cells secrete cytokines, whereby they play an important immunoregulatory role and are able to differentiate into CTLs [6].

When assessing the content of T-lymphocytes with $\gamma \delta$ TCR-receptor, we found an increase in the percentage of this subpopulation of lymphocytes both in the entire group of patients with RMS and in patients with unfavorable localization of the primary tumor. It is possible that an increase in the number of $\gamma \delta \mathrm{TCR}-\mathrm{lymph}-$ ocytes has an inhibitory effect on the spread of RMS.

These data obtained by us in the analysis of the content of $\gamma \delta \mathrm{TCR}-\mathrm{T}-\mathrm{lymph}$ ocytes require further evaluation and more in-depth study in order to identify their clinical and prognostic value, influence on the course of the tumor process and treatment results.

When assessing the number of T-lymphocytes depending on the location of the primary tumor, we found that the CD3+HLA-DR+ subpopulation was significantly higher in both groups of patients, mainly due to an increase in CTL (CD8+ HLA-DR+ cells). It is interesting to note that in patients with favorable localization of the tumor, this subpopulation was higher in absolute value, and in patients with unfavorable localization-in relative value. In both cases, we can explain the increase in the number of $\mathrm{CD} 3+\mathrm{T}$ cells by the effective presentation of antigenic determinants to T-lymphocytes with their subsequent activation and clonal proliferation [6], as evidenced by the expression of the activation molecule HLA-DR. But in patients with favorable localization, more active antigenic 
stimulation of T cells by sarcoma is quite possible. Otherwise, the comparison of the number of $\mathrm{CD} 3+\mathrm{T}$ cells of patients with RMS with control values, as well as within different clinical groups, did not reveal significant differences.

The entire population of CTL (CD3+ CD8+ T cells) was increased in patients with favorable tumor localization (in absolute terms) compared to those in whom the localization of the primary tumor was regarded as unfavorable. We believe that the increase in the percentage of CD3+ CD8+ T cells in patients with RMS reflects, in the absence of a "tumor counterattack", a significant activation and proliferation of CTL, which reaches optimal values with a favorable localization of the primary tumor. Such observations regarding the infiltration of the tumor by CD8+ T-lymphocytes are considered as a prognostic factor for the course of oncological diseases [12].

Comparison of absolute CD5+ values $\mathrm{T}$ cells showed an increase in their number in patients with a favorable localization of the primary tumor, which may reflect a higher proliferative response of the patient's immune system to the development of the tumor and/or the absence of cytotoxic effects of sarcoma cells by the type of tumor "counterattack", which generally contributes to the accumulation of $\mathrm{T}$ cells with this marker. The role of $\mathrm{CD} 5+\mathrm{T}$-lymphocytes in the course of RMS has yet to be explored in more clinical examples.

The CD3+ subpopulation of $\mathrm{T}$ cells, which has a marker of cell adhesion/activetion of $\mathrm{CD} 2+$, was significantly higher in absolute value in patients with RMS with favorable tumor localization. It is possible that the biological features of the sarcoma of this localization contribute to the optimal activation of effector T-lymphocytes and the appearance of the CD2 adhesion marker on their surface, which promotes the penetration of $\mathrm{T}$ cells into the tumor tissue. This assumption also requires further study.

CD3+ T cells with the expression of the costimulatory molecule CD28+, which is necessary for the effective presentation of the antigen to the T-lymphocyte [6], were significantly increased only in patients with parameningeal localization of the tumor, both in relative and absolute values. A decrease in the percentage of this $\mathrm{T}$ cell subpopulation was observed in patients who had an alveolar histological variant of the tumor. It is possible that in these clinical groups there are significant differences in the mechanisms of antigen presentation to T-lymphocytes. In the first case, most likely, there is an effective presentation of the antigen with clonal proliferation and accumulation of $\mathrm{CD} 3+\mathrm{CD} 28+\mathrm{T}$ cells as a result, and in the second-an ineffective presentation with subsequent energy of T-lymphocytes as a manifestation of one of the ways of escaping the tumor from immune surveillance [13]. This assumption can be confirmed or excluded in an in-depth study of the features of the presentation of Antigen-Presenting Cells (APC) of antigenic determinants in RMS. The clinical and prognostic significance of the changes in the BM subpopulation composition of patients with RMS within these clinical groups remains to be determined when studying the role of CD3+ CD28+ T cells during the tumor process and the results of treatment in a larger number of clini- 
cal examples.

$\mathrm{CD} 3+\mathrm{CD} 56+$ are immunological markers of NKT cells that are present in BM and play an active role in the antitumor immune response [6] [14]. When stimulated, NKT cells produce a large number of T-helper cytokines of both types, which plays a significant role in the modulation of other immune effector cells (CD4+ and CD8+ T cells, NK cells and B cells involved in Th-1 and Th- 2 immune responses) [15]. One of the interesting properties of the NKT cell population is their paradoxical functions in various pathological conditions, including in malignant tumors and autoimmune diseases [15]. The functions of NKT cells can be useful or harmful in various cases, which may be due to the balance between immune responses moved towards Th-1 or Th-2 types [15]. However, we did not find significant differences in the number of NKT cells depending on the localization of the primary tumor and its histological structure.

In our work, we analyzed the number of CD57+ CTL (CD8+ CD57+ T cells) in patients with RMS, which appear as a result of the accumulation of antigen-specific oligoclonal $\mathrm{T}$ cells in the late stages of differentiation during chronic antigenic stimulation [16]. According to their functional properties, such cells are characterized as highly cytotoxic [16]. It was also shown that the expression of CD57+ strongly correlates with the simultaneous expression of granzymes and perforin in CD8+ T cells, and the expression of these cytolytic molecules in CD8+ CD57+ T cells is significantly higher than in their CD8+ CD57- precursors [16]. However, when comparing the level of this subpopulation in the analyzed clinical groups with the control values, we did not find significant differences either in relative amounts or in absolute values. This result is most likely a sign of weak immunogenicity of RMS and a feature of the immune response in this type of sarcoma, as was proved in the work of Tanzarella et al. [11].

Another important subpopulation of $\mathrm{T}$ cells-effectors of antitumor immunity are helper T-lymphocytes (CD3+ CD4+ T cells) [6]. When estimating the number of this subpopulation, we obtained interesting results. Thus, the content of $\mathrm{CD} 3+\mathrm{CD} 4+\mathrm{T}$ cells of $\mathrm{BM}$ was significantly reduced compared to the control values in patients with RMS with an unfavorable localization of the primary tumor and an unfavorable (alveolar) histological variant of the tumor. We can assume that in the analyzed cases, it is possible to inhibit the proliferation of $\mathrm{T}$ helper cells and/or eliminate them by sarcoma cells in the "tumor counterattack" type with the participation of the Fas-FasL system [13]. To confirm or refute these hypotheses, a detailed study of the biological features of the RMS cells and the mechanisms of their interaction with the patient's immune system is required.

The $\mathrm{T}$ cell adhesion molecule Leu8 (CD62L) plays an important role in the penetration of lymphocytes into the foci of inflammation and other tissue damage, as it promotes the attachment of lymphocytes to the vessel wall, subsequent "rolling" and extravasations of $\mathrm{T}$ cells into the surrounding tissues (in our case, in BM) [6]. Comparison of the number of T-helper cells with the Leu8 adhesion 
molecule (Leu8+ CD4+ T cells) in patients with RMS with control values revealed a decrease in the percentage of this subpopulation both in the entire group of RMS patients and in the compared clinical groups: in patients with unfavorable localization of the primary tumor (as opposed to patients with favorable localization), and in patients with a favorable embryonic histological variant in comparison with the alveolar variant. It is possible that the inhibition of the Leu8+ $\mathrm{CD} 4+\mathrm{BM}$ subpopulation is a characteristic feature of the immunodeficiency state in RMS. It is obvious that a decrease in the percentage of CD3+ CD4+ T-helper cells together with a decrease in the percentage of Leu8 $+\mathrm{CD} 4+\mathrm{T}$ cells in patients with RMS may indicate a simultaneous inhibition of the T-helper link and the process of extravasations of T-helper cells in the tissue. The decrease in the accumulation of T-helper cells in BM may reflect a new, previously undescribed type of immunodeficiency-the inability of effector cells of the immune system to accumulate both in the lesion site and in the central immunoregulatory organ-BM.

In our work, we also analyzed the content of B-1a-a subpopulation of B-lymphocytes (CD19+ CD5+ cells) in the BM of patients with RMS to assess the state of B-lymphopoiesis. As is currently known, the function of these B-lymphocytes is a rapid response to widespread pathogens (mainly bacteria) that enter the body in the form of IgM synthesis, as well as the synthesis of polyspecific natural immunoglobulins present in the blood and body fluids regardless of the presence of the antigen [6]. The absolute content of CD19+ CD5+ B cells was higher in patients with RMS with favorable prognostic localization of the tumor compared to patients with unfavorable localization; the relative content of B1a cells did not differ between these clinical groups. It is possible that pre-existing changes in the patient's immune system allow for the development of an RMS clone of a particular localization, or the appearance and proliferation of a malignant clone differently affects the humoral and cellular components of immunity, depending on the clinical features of the tumor [17]. These assumptions are extremely interesting for further study in terms of the interaction of the tumor and the human body.

Summarizing the results obtained, we attempted to present the relationship of changes in the level of BM lymphocyte subpopulations with risk factors for RMS and to reflect the influence or cause of this phenomenon from the point of view of the "tumor-immunity" system (Table 10).

Table 10. The relationship of changes in the level of BM lymphocyte subpopulations with risk factors for RMS and the "tumorimmunity" system.

\begin{tabular}{ccccc}
\hline Subpopulations & Value & $\begin{array}{c}\text { Change } \\
\text { vector }\end{array}$ & Risk factors & Presumptive reason \\
\hline CD3+ & abs & $\uparrow$ & Favorable tumor localization & Clonal proliferation of T cells after antigen recognition on \\
CD3+ HLADR+ & $\%$ & $\uparrow$ & ALL patients & Effective presentation of tumor antigens to T-lymphocytes \\
\hline
\end{tabular}




\section{Continued}

\begin{tabular}{|c|c|c|c|c|}
\hline CD8+ HLADR+ & $\%$ & $\uparrow$ & ALL patients & $\begin{array}{l}\text { Effective presentation of tumor antigens to cytotoxic } \\
\text { T-lymphocytes }\end{array}$ \\
\hline $\mathrm{CD} 5+$ & abs & $\uparrow$ & Favorable tumor localization & Favorable prognostic factor (?) \\
\hline $\mathrm{CD} 3+\mathrm{CD} 2+$ & abs & $\uparrow$ & Favorable localization & Active $\mathrm{T}$ cell penetration in $\mathrm{BM}$ \\
\hline $\mathrm{CD} 3+\mathrm{CD} 28+$ & $\%$ & $\uparrow$ & Parameningeal localization of RMS & $\begin{array}{l}\text { Efficient antigen presentation with clonal proliferation and } \\
\text { accumulation of } \mathrm{CD} 3+\mathrm{CD} 28+\end{array}$ \\
\hline $\mathrm{CD} 3+\mathrm{CD} 28+$ & $\%$ & $\downarrow$ & Alveolar RMS & $\begin{array}{c}\text { Anergy of T-lymphocytes, tumor evasion from immune } \\
\text { surveillance }\end{array}$ \\
\hline $\mathrm{CD} 3+\mathrm{CD} 56+$ & $\begin{array}{l}\% \\
\text { abs }\end{array}$ & $\begin{array}{c}\text { Not } \\
\text { changed }\end{array}$ & All groups & Low immunogenicity of RMS \\
\hline $\mathrm{CD} 3+\mathrm{CD} 4+$ & $\%$ & $\downarrow$ & $\begin{array}{c}\text { Unfavorable localization } \\
\text { Alveolar variant }\end{array}$ & $\begin{array}{l}\text { Inhibition of the proliferation of T-helpers or } \\
\text { "tumor counterattack" }\end{array}$ \\
\hline Leu8+ CD4+ & $\%$ & $\downarrow$ & All RMS patients & Immunodeficiency in patients with RMS \\
\hline$\alpha \beta \mathrm{TCR}$ & abs & $\uparrow$ & Favorable localization of the primary tumor & Favorable prognostic factor (?) \\
\hline$\gamma \delta \mathrm{TCR}$ & abs & $\uparrow$ & Favorable localization of the primary tumor & Favorable prognostic factor (?) \\
\hline$\gamma \delta \mathrm{TCR}$ & $\%$ & $\uparrow$ & All RMS patients & Containing the spread of RMS \\
\hline $\mathrm{CD} 19+\mathrm{CD} 5+$ & abs & $\uparrow$ & Favorable localization & Influence of RMS on the humoral link of immunity \\
\hline
\end{tabular}

\section{Conclusions}

As is currently known, the biology of RMS cells determines the histological structure and localization of the tumor. We believe that each clinical-age group of patients may have its own individual immunological characteristics and features of cell killing reactions against a tumor. Based on the results of the studies carried out, it is possible that new immunological factors for prognosis, clinical course and treatment of patients with RMS will be determined, depending on the histological structure of the tumor and its localization.

Summarizing our results, it can be noted that the revealed increase in the content of CD3+ T cells (in particular, a subpopulation of CD8+ CTLs) is a quite expected result, which reflects the response of the immune system to tumor development: effective presentation of tumor antigens, their recognition and activation of naive T-ymphocyte with following clonal proliferation. An increase in the content of a special $\gamma \delta \mathrm{TCR}-\mathrm{T}$ cell subpopulation is an interesting finding that expands our understanding of the features of the antitumor immune response in this neoplasia and the significance of which remains to be clarified. Changes in the expression of activation and co-stimulatory molecules on the surface of CTLs and T-helpers may have an important clinical and prognostic value in terms of assessing the functional efficiency of the cellular link of the patient's immune system.

But on the whole, the data we obtained can be considered indicative and regarded as starting points for further research of the peculiarities of the subpopulation composition of BM in patients with RMS using a larger number of clinical 
examples. Our results of studying the characteristics of the immune response of the patient's organism to the development of RMS can contribute to the development and implementation of immunotherapy protocols in order to increase the overall and disease-free survival of patients.

\section{Conflicts of Interest}

The authors declare no conflicts of interest regarding the publication of this paper.

\section{References}

[1] Yohe, M.E., Heske, C.M., Stewart, E., et al. (2019) Insights into Pediatric Rhabdomyosarcoma Research: Challenges and Goals. Pediatric Blood \& Cancer, 66, e27869. https://doi.org/10.1002/pbc.27869

[2] Gurria, J.P. and Dasgupta, R. (2018) Rhabdomyosarcoma and Extraosseous Ewing Sarcoma. Children, 5, 165. https://doi.org/10.3390/children5120165

[3] Yang, L.M., Takimoto, T. and Fujimoto, J. (2014) Prognostic Model for Predicting Overall Survival in Children and Adolescents with Rhabdomyosarcoma. BMC Cancer, 14, 654. https://doi.org/10.1186/1471-2407-14-654

[4] Feuerer, M., Beckhove, P., Garbi, N., et al. (2003) Bone Marrow as a Priming Site for T-Cell Responses to Blood-Borne Antigen. Nature Medicine, 9, 1151-1157. https://doi.org/10.1038/nm914

[5] Zhao, E., Xu, H., Wang, L., et al. (2012) Bone Marrow and the Control of Immunity. Cellular \& Molecular Immunology, 9, 11-19. https://doi.org/10.1038/cmi.2011.47

[6] Khaitov, R.M. (2018) Immunology. Geotar-Media, Moscow.

[7] Koh, B.I. and Kang, Y.B. (2012) The Pro-Metastatic Role of Bone Marrow-Derived Cells: A Focus on MSCs and Regulatory T Cells. EMBO Reports, 13, 412-422. https://doi.org/10.1038/embor.2012.41

[8] Madhumathi, D.S., Premalata, C.S., Devi, V.L., et al. (2007) Bone Marrow Involvement at Presentation in Pediatric Non-Haematological Small round Cell Tumours. Indian Journal of Pathology \& Microbiology, 50, 886-889.

[9] Pantel, K. and Alix-Panabieres, C. (2014) Bone Marrow as a Reservoir for Disseminated Tumor Cells: A Special Source for Liquid Biopsy in Cancer Patients. BoneKEy Reports, 3, 584. https://doi.org/10.1038/bonekey.2014.79

[10] Simon-Keller, K., Paschen, A., Eichmuller, S., et al. (2010) Adoptive T-Cell Therapy of Rhabdomyosarcoma. Pathologie, 31, 215-220. https://doi.org/10.1007/s00292-010-1344-8

[11] Tanzarella, S., Lionello, I., Valentinis, B., et al. (2004) Rhabdomyosarcomas Are Potential Target of MAGE-Specific Immunotherapies. Cancer Immunology, Immunotherapy, 53, 519-524. https://doi.org/10.1007/s00262-003-0484-6

[12] Kiselevsky, M.V., Vlasenko, R.Y., Zabotina, T.N. and Kadagidze, Z.G. (2019) Prognostic Significance of Tumor-Infiltrating Lymphocytes. Immunology, 40, 73-82.

[13] Igney, F.H. and Krammer, P.H. (2002) Immune Escape of Tumors: Apoptosis Resistance and Tumor Counterattack. Journal of Leukocyte Biology, 71, 907-920.

[14] Dean, J., McCarthy, D., Lawler, M., et al. (2005) Characterization of NKR + T-Cell Subsets in Normal Human Bone Marrow: Implications for Immunosurveillance of Neoplasia. Clinical Immunology, 114, 42-51. 
https://doi.org/10.1016/j.clim.2004.08.017

[15] Lin, H., Nieda, M., Rosenkov, V. and Nicol, A.J. (2006) Analysis of the Effect of Different NKT Cell Populations on the Activation of CD4 and CD8 T Cells, NK Cells, and B Cells. Experimental Hematology, 34, 289-295.

https://doi.org/10.1016/j.exphem.2005.12.008

[16] Strioga, M., Pasukoniene, V. and Characiejus, D. (2011) CD8(+) CD28(-) and CD8(+) CD57(+) T Cells and Their Role in Health and Disease. Immunology, 134, 17-32. https://doi.org/10.1111/j.1365-2567.2011.03470.x

[17] Kolbatskaya, O.P., Gorbunova, T.V., Polyakov, V.G., Tupitsyn, N.N., Popa, A.V., Serebryakova, I.N., Timoshenko, V.V. and Shvedova, T.V. (2016) Comparative Characteristics of Subpopulations of Bone Marrow Lymphocytes in Children with Acute Leukemia, Rhabdomyosarcoma and Ewing's Sarcoma. Oncopediatrics, 3, 91-106. https://doi.org/10.15690/onco.v3i2.1543 